\title{
Optimal Supply of a Depletable Resource with a Backstop Technology: Heal's Theorem Revisited
}

\author{
SHMUEL S. OREN \\ University of California, Berkeley, California \\ STEPHEN G. POWELL \\ Wang Laboratories, Inc., Lowell, Massachusetts
}

(Received April 1983; revised January 1984; accepted April 1984)

\begin{abstract}
Heal's theorem states that if the extraction cost of a depletable resource increases with cumulative extraction, and if a backstop technology exists, the user cost of the depletable resource declines to zero at the date of exhaustion. In this paper, we first present a simple method for proving this proposition, using a social planning model that determines the optimal rates both of extraction of the depletable resource and of production of the backstop technology. We then present two examples that show how this method can be used to solve more difficult problems in the theory of resource economics. The first example involves learning-by-doing in the backstop sector; that is, backstop costs decline with cumulative production. The second example involves uncertainty of backstop costs.
\end{abstract}

$\mathrm{I}^{\mathrm{N}}$ N A 1976 article in the Bell Journal, Geoffrey Heal established the following important proposition concerning the role of extraction costs in determining the socially optimal price of a depletable resource. Assume the resource is available in infinite amounts, but that its extraction cost rises with cumulative extraction. The resource cost is bounded from above by a so-called "backstop" technology, which provides unlimited amounts of the resource at a constant cost. Heal claimed that in such a world the socially optimal price for the resource must start out well above the marginal extraction cost of the depeletable resource, and move toward it as cumulative extraction grows. Equivalently, the user cost of the resource is initially high, but declines to zero at the instant of transition to the backstop technology. This result contrasts sharply with the case in which extraction costs are constant and the total stock of the depletable resource is finite. With these alternative assumptions, the socially optimal price starts out close to the cost of extraction and rises steadily above it as the resource stock is consumed. The user cost of a constant-cost depletable resource reaches its maximum at the transition to the backstop.

Subject classification: 131 Heal's theorem revisited, 473 optimal supply of a depletable resource. 
Heal's statement of these results has been sharpened in several important ways by subsequent authors. Clark [1978] pointed out that the user cost need not necessarily decline to zero monotonically, in particular if the change in the marginal extraction cost with cumulative extraction is initially small. Hanson [1980] then established an important relationship between the rate of change of user costs and the curvature of the resource price path. In particular, Hanson showed that the price path must be concave as it approaches the level of backstop costs, and the user cost must be decreasing as well. However, it is perfectly possible (again, depending on the time rate of change of marginal extraction costs) for the price path to be convex and user costs to be increasing over an earlier time interval. In this paper, we will denote by "Heal's theorem" the basic result that the user cost of depletable resources goes to zero at the transition to the backstop.

In Figure 1 we illustrate the differences between the constant-cost and increasing-cost formulations. If the unit cost of the depletable resource is constant at value $d$, and the backstop is available at a cost $c>d$, the socially optimal price starts out close to $d$ and rises until it reaches $c$ at the transition date $T$ (Figure 1a). The user cost component of the price, which measures the cost to society (in excess of the extraction cost) of consuming a unit of the finite stock, rises monotonically as long as the depletable resource is being consumed. Because the stock of the depletable resource is finite, consuming a unit today reduces the quantity available for later consumption. This hidden cost of consumption is reflected in the user cost, and it naturally rises as the remaining stock declines toward zero. In Figure $1 \mathrm{~b}$ we illustrate the alternative case, in which the resource stock is unlimited but the marginal cost of extraction rises with cumulative extraction. As in the previous case, the price of the

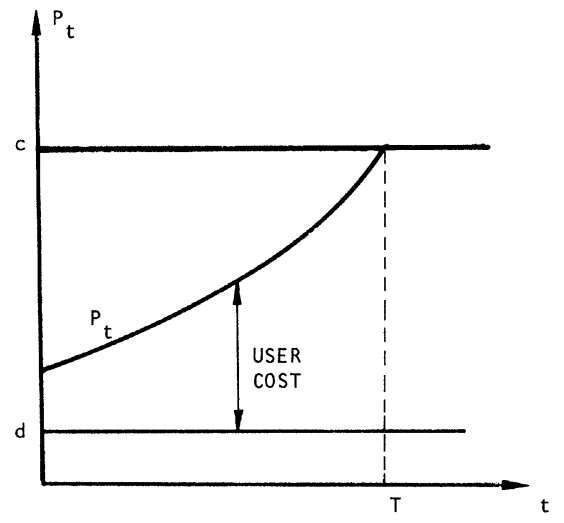

a. CONSTANT COST

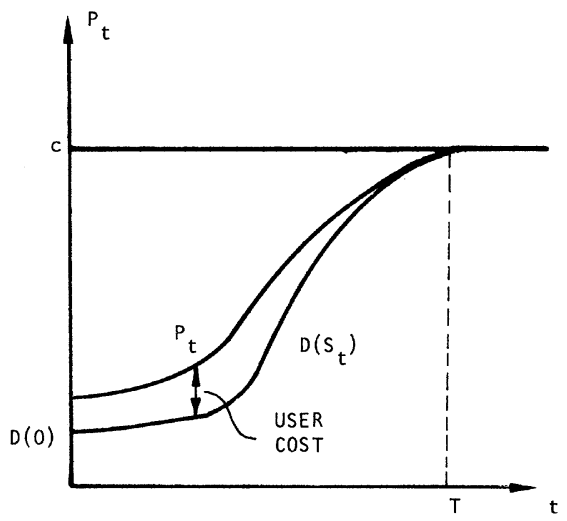

b. RISING COST

Figure 1. User costs for depletable resources. 
resource rises monotonically to the backstop cost at the transition date. However, the marginal cost of extraction $D\left(S_{t}\right)$ is rising along with cumulative extraction $S_{t}$. And, as Figure 1 shows, as the price nears the level of backstop costs, the user cost is declining. At the transition date, price and marginal extraction cost are equal: the user cost has reached zero. In this case the user cost reflects the fact that consuming a unit of resource today pushes society up the cost curve, and in effect makes all future units more expensive to extract. This shadow cost falls over time simply because the number of units of the depletable resource left to consume before society switches to the backstop is shrinking.

Heal's proof of this result is based on an optimal growth model that combines the reproducible capital and the depletable resource to produce a single homogeneous output. Society must choose not only the rate at which resources are used in production, but also the division of current output between consumption and investment. However, the same result can be proven using a simpler model that directly chooses the optimal rates of consumption of depletable and backstop resources. The method of proof developed here is both simpler and more intuitive than that used in Heal's original paper. Moreover, this method generalizes easily to more complex problems in the theory of depletable resources, making possible proofs of some results which have previously been assumed. Our purpose in this paper is to develop this simpler proof of Heal's theorem, and to illustrate its application in several more difficult problems.

The paper is organized as follows. In Section 1, we develop a simple social planning model with two resources, one depletable and the other the backstop. This discussion develops the mathematical solution to this optimal control problem in detail, and shows that Heal's result follows naturally from a transversality condition. Then, in Section 2, we show how the same general method can solve a more difficult problem, in which backstop costs decline with cumulative production as a result of learning-by-doing. The solution to this problem requires a theorem on the value of an optimal control problem with discounted objective, which is proven in an appendix. In Section 3 we study another generalization of the basic model, one in which backstop costs are uncertain. Again, the same method of attack provides a more thorough analysis than has been available to date. The paper closes with a brief summary.

\section{THE BASIC METHOD AND PROOF OF HEAL'S THEOREM}

Consider the problem of choosing the socially optimal rates of consumption of two resources, one a depletable resource whose costs rise with cumulative extraction, the other a backstop technology with constant costs. Let $S_{t}$ represent cumulative extraction of the depletable resource, and $D\left(S_{t}\right)$ the marginal cost of extraction. We will assume that the available supply of the depletable resource is unlimited, and that 
$D(\cdot)$ is strictly monotonically increasing and unbounded. The backstop technology provides an unlimited supply of the resource at a constant unit cost $c>D(0)$.

As is standard, we will assume the existence of a concave utility functions $u(\cdot)$, which measures the gross value to society of consuming the resource. We will further assume that $u^{\prime}(0)>c$. The social planner's problem is to determine extraction (and consumption) rates of the two resources that maximize the present value of utility net of extraction costs. The problem can be formally stated as

$$
\operatorname{Max}_{\left\{x_{t}, y_{t}\right\}} \int_{0}^{\infty} e^{-r t}\left[u\left(x_{t}+y_{t}\right)-D\left(S_{t}\right) x_{t}-c y_{t}\right] d t
$$

subject to

$$
\begin{aligned}
\dot{S}(t) & =x(t) ; \quad S(0)=0 \\
x(t) & \geq 0 \\
y(t) & \geq 0,
\end{aligned}
$$

where

$$
\begin{aligned}
x(t) & =\text { rate of extraction of the depletable resource } \\
y(t) & =\text { rate of production of the backstop resource } \\
S(t) & =\text { cumulative extraction of the depeletable resource } \\
c & =\text { cost of the backstop resource } \\
u(\cdot) & =\text { social utility function } \\
r & =\text { social discount rate. }
\end{aligned}
$$

First, we show that the solution to (1) involves an initial phase where extraction of the depletable resource is positive and backstop production is zero, followed by a phase where only backstop production is positive. This result allows us to recast (1) as a finite horizon, free end-time control problem in which the single control variable is the extraction rate of the depletable resource. We then show that Heal's theorem follows naturally from the transversality condition that links the rates of extraction of the depletable resource and the backstop at the transition date.

The Hamiltonian for problem (1) is given by

$$
H=u\left(x_{t}+y_{t}\right)-D\left(S_{t}\right) x_{t}-c y_{t}+\lambda_{s}(t) x_{t},
$$

where the adjoint variable $\lambda_{s}(t)$ can be interpreted as a shadow price that measures the sensitivity of the objective to changes in cumulative extraction $S_{t}$. The three necessary conditions for optimality that follow from the maximum principle are

$$
\begin{aligned}
u^{\prime}\left(x_{t}+y_{t}\right)-D\left(S_{t}\right)+\lambda_{s}(t) & =0 \text { if } \quad x_{t}>0 \\
& \leq 0 \text { if } \quad x_{t}=0
\end{aligned}
$$




$$
\begin{aligned}
u^{\prime}\left(x_{t}+y_{t}\right)-c & =0 \quad \text { if } \quad y_{t}>0 \\
& \leq 0 \quad \text { if } \quad y_{t}=0 ; \\
\dot{\lambda_{s}}(t) & =r \lambda_{s}(t)+D^{\prime}\left(S_{t}\right) x_{t} .
\end{aligned}
$$

We first establish a preliminary result.

Proposition 1. In the optimal solution to (1), both resources cannot be produced simultaneously over any finite interval.

Proof. Differentiating (3) and combining the result with (5) yields $\dot{x}_{t}+\dot{y}_{t}=r\left[u^{\prime}\left(x_{t}+y_{t}\right)-D\left(S_{t}\right)\right] / u^{\prime \prime}\left(x_{t}+y_{t}\right)$ when $x_{t}>0$. On the other hand, from (4) we have $u^{\prime}\left(x_{t}+y_{t}\right)=c$ and consequently $\dot{x}_{t}+\dot{y}_{t}=0$ when $y_{t}>0$. Since $D\left(S_{t}\right)$ is strictly increasing when $x_{t}>0$, conditions (3) and (4) for $x_{t}>0$ and $y_{t}>0$ cannot hold simultaneously on any finite time interval.

Our assumption on $D(\cdot)$ implies the existence of some finite $\bar{S}$ satisfying $D(\bar{S})=c, D(S)<c$ for $S<\bar{S}$ and $D(S)>c$ for $S>\bar{S}$. In other words, there is some finite amount of the depletable resource that is cheaper than any unit of the backstop. Since the objective in (1) is linear in costs, it is optimal to extract depletable energy as long as its cost is less than the backstop. Furthermore, due to discounting and in view of Proposition 1, depletable energy should be used before any backstop energy is produced. These observations can be summarized as follows:

Proposition 2. The solution to (1) requires that the depletable resource be consumed over the finite interval $[0, T]$ up to the point where the marginal extraction cost of the depletable resource equals the backstop cost. After T, only the backstop resource is used.

In view of Proposition 2, we may rewrite the original problem (1) as an equivalent finite-horizon control problem. In this problem, the planner seeks the optimal rate of extraction of the depletable resource and a shutdown date at which the transition to the backstop occurs. Formally we have

$$
\operatorname{Max}_{\left\{x_{t}\right\}, T} \int_{0}^{T} e^{-r t}\left[u\left(x_{t}\right)-D\left(S_{t}\right) x_{t}\right] d t+\Phi(T)
$$

subject to

$$
\dot{S}(t)=x_{t} ; \quad S(0)=0, \quad S(T)=\bar{S}
$$

where

$$
\begin{aligned}
x(t) \geq & 0, \\
\Phi(T)= & \operatorname{Max}_{\left\{y_{t}\right\}} \int_{T}^{\infty} e^{-r t}\left[u\left(y_{t}\right)-c y_{t}\right] d t \\
& \text { subject to } y(t) \geq 0 .
\end{aligned}
$$


The function $\Phi(T)$ can be interpreted as a terminal reward that equals the net present value of social utility derived from backstop production. To obtain an explicit expression for $\Phi(T)$, we need to know the optimal backstop production rate $y_{t}$. We note, however, that this optimal $y_{t}$ can be determined pointwise by solving the static problem

$$
\operatorname{Max}_{\{y(t)\}}\left[u\left(y_{t}\right)-c y_{t}\right], \quad \text { subject to } y(t) \geq 0 .
$$

Thus, the optimal $y_{t}$ is constant and must satisfy the first order condition $u^{\prime}\left(y^{*}\right)=c$, i.e., marginal utility equals (marginal) cost. Substituting $y_{t}=y^{*}$ in the above expression for $\Phi(T)$ yields:

$$
\Phi(T)=(1 / r) e^{-r T}\left[u\left(y^{*}\right)-c y^{*}\right] .
$$

We next consider the solution for problem (6). The Hamiltonian for this problem is

$$
H=u\left(x_{t}\right)-D\left(S_{t}\right) x_{t}+\lambda_{s}(t) x_{t}
$$

and by the maximum principle we obtain the necessary conditions:

$$
\begin{aligned}
u^{\prime}\left(x_{t}\right)-D\left(S_{t}\right)+\lambda_{s}(t) & =0 \quad \text { if } \quad x_{t}>0 \\
& \leq 0 \quad \text { if } \quad x_{t}=0 . \\
\dot{\lambda}_{s}(t) & =r \lambda_{s}(t)+D^{\prime}\left(S_{t}\right) x_{t} . \\
H(T) & =-e^{r T}(\partial \Phi(T) / \partial T) .
\end{aligned}
$$

Equation 11 is the transversality condition that applies to a free endtime control problem. Heal's result, which is stated in the following proposition, follows from this condition.

Proposition 3 (Heal's Theorem). The shadow cost on cumulative extraction of depletable energy goes to zero at the instance of transition to the backstop, i.e.,

$$
\lambda_{s}(T)=0 .
$$

Proof. From (11) and (7) we have

$$
\begin{aligned}
H(T) & =-e^{r T}(\partial \Phi(T) / \partial T) \\
& =-e^{r T}\left[e^{-r T}\left(u\left(y^{*}\right)-c y^{*}\right)\right] \\
& =u\left(y^{*}\right)-c y^{*} .
\end{aligned}
$$

But from (8) and (9),

$$
\begin{aligned}
H(T) & =u\left(x_{T}\right)-D\left(S_{T}\right) x_{T}+\lambda_{s}(T) x_{T} \\
& =u\left(x_{T}\right)-u^{\prime}\left(x_{T}\right) x_{T} .
\end{aligned}
$$


Thus,

$$
u\left(x_{T}\right)-u^{\prime}\left(x_{T}\right) x_{T}=u\left(y^{*}\right)-c y^{*} .
$$

Now, since $u(\cdot)$ is concave, the only solution for $x_{T}$ is $x_{T}=y^{*}$. But then, since $D\left(S_{T}\right)=c$,

$$
\lambda_{s}(T)=D\left(S_{T}\right)-u^{\prime}\left(x_{T}\right)=c-u^{\prime}\left(y^{*}\right)=0 .
$$

We conclude this section by summarizing the solution to the social planning problem presented earlier. In the initial phase, from 0 to $T$, only depletable energy is produced. From (9) and (10) we can show that

$$
\dot{x}_{t}=r\left[u^{\prime}\left(x_{t}\right)-D\left(S_{t}\right)\right] / u^{\prime \prime}\left(x_{t}+y_{t}\right) .
$$

Since $u^{\prime}\left(x_{t}\right)>D\left(S_{t}\right)$, and $u^{\prime \prime}(\cdot)<0$, we can conclude that $\dot{x}_{t}<0$. Thus, throughout the first phase, depletable resource production is declining, and marginal utility is rising. Since $x_{T}=y^{*}$, total resource use is continuous across the transition from depletable to backstop use at $T$. As a consequence, marginal utility rises smoothly to the cost of the backstop and remains constant at that level afterward. Finally, we know from Proposition 3 that the shadow $\operatorname{cost} \lambda_{s}(t)$ goes to zero at $T$. Therefore, as we illustrate in Figure 1b, the gap between the current extraction cost and the marginal value of the resource, which is equivalent to its price, declines toward zero as the transition date approaches. As a final comment, we want to stress that the sharp transition to the backstop is to a large extent a result of the simplifying assumption of the model. Kemp and Long [1980] show that this result need not hold in a general equilibrium framework. They essentially argue that as the rate of production of depletable resources declines over time, the optimal policy idles more factors of production. Employing these factors in backstop production raises welfare over the alternative of delaying backstop production until the depletable resource is exhausted.

\section{LEARNING-BY-DOING}

By assumption, the backstop technology relies on a superabundant resource base. Consequently, its long-run costs should be relatively constant, and not rise as is typical of depletable resources. On the other hand, the costs of early units of backstop production are likely to decline, as more efficient production processes are discovered and practical experience accumulates. This "learning-by-doing" effect is widespread in new industries, and can account for substantial reductions in production costs. In this section we analyze a planning model that incorporates learning-by-doing in the backstop sector.

Let $R_{t}$ represent cumulative production of the backstop resource, and 
$E\left(R_{t}\right)$ the marginal production cost. We will assume $E\left(R_{t}\right)$ is convex and declines toward an asymptote $E_{\infty}$, as shown in Figure 2. The planner's problem for this generalization of (1) can be stated as follows.

$$
\begin{array}{cl}
\operatorname{Max}_{\left\{x_{t}, y_{t}\right\}} \int_{0}^{\infty} e^{-r t}\left[u\left(x_{t}+y_{t}\right)-D\left(S_{t}\left(x_{t}-E\left(R_{t}\right) y_{t}\right] d t\right.\right. \\
\text { subject to } \quad \dot{S}(t)=x(t) ; \quad S(0)=0 \\
\dot{R}(t)=y(t) ; \quad R(0)=0 . \\
x(t) \geq 0 \\
y(t) \geq 0 .
\end{array}
$$

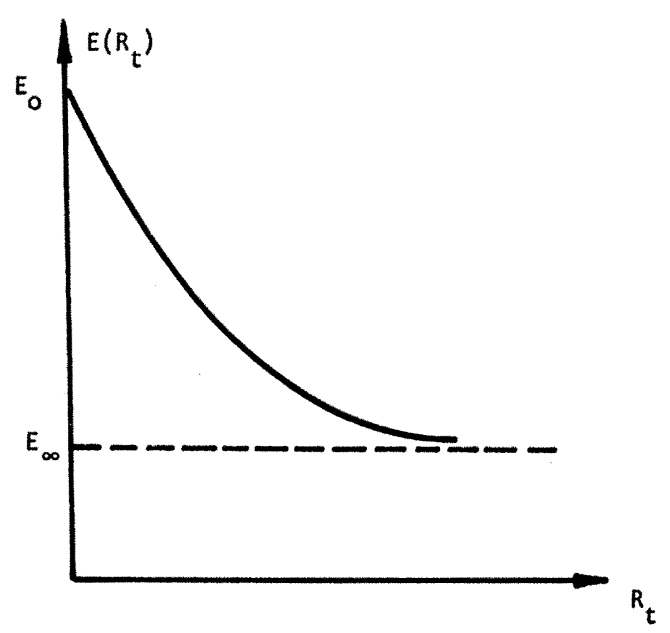

Figure 2. Backstop production costs.

Since the solution to (13) parallels that to (1) in most respects, we will leave out many of the details.

First, using essentially the same technique as in the proof of Proposition 1 , we can show that $x_{t}$ and $y_{t}$ cannot both be positive over any finite interval. And again, if any of the depletable resource is cheaper to extract than the first unit of the backstop is to produce, then the depletable resource will be produced first. Finally, there can be only one transition; that is, once backstop production begins, the depletable resource is never extracted again.

These considerations allow us to rewrite (13) as the finite-horizon 
problem

$$
\operatorname{Max}_{\left\{x_{t}\right\}, T} \int_{0}^{T} e^{-r t}\left[u\left(x_{t}\right)-D\left(S_{t}\left(x_{t}\right] d t+\Phi(T)\right.\right.
$$

subject to $\quad \dot{S}(t)=x(t) ; \quad S(0)=0$

$$
x(t) \geq 0
$$

where

$$
\Phi(T)=\operatorname{Max}_{\left\{y_{t}\right\}} \int_{T}^{\infty} e^{-r t}\left[u\left(y_{t}\right)-E\left(R_{t}\right) y_{t}\right] d t
$$

$$
\begin{aligned}
\text { subject to } \quad \dot{R}(t) & =y(t) ; \quad R(T)=0 . \\
y(t) & \geq 0 .
\end{aligned}
$$

Powell [1983] derives several interesting properties of the solution to the second phase of (14). It is shown, for example, that the optimal rate of backstop production increases over time. Moreover, the marginal utility associated with $y_{t}$ is always bounded above by $E\left(R_{t}\right)$, and approaches $E_{\infty}$ asymptotically. With costs declining as a function of cumulative production, the user cost associated with current production is negative. In other words, backstop production is pushed to a point where marginal utility is less than current production costs, since there is a positive benefit from moving down the cost curve. But for our purposes, the most interesting aspect of the second phase is that we can express $\Phi(T)$ as a function of the initial rate of backstop production $y_{T}$. Thus, we have the following result.

\section{Proposition 4.}

$$
\Phi(T)=(1 / r) e^{-r T}\left[u\left(y_{T}\right)-u^{\prime}\left(y_{T}\right) y_{T}\right]
$$

Proof. The proof rests on the theorem and corollaries proved in the Appendix. These results allow us to express the value of an optimal control problem with discounted objective in terms of the Hamiltonian at the initial and terminal times. The Hamiltonian for the second phase is

$$
H(T)=u\left(y_{t}\right)-E\left(R_{t}\right) y_{t}+\lambda_{r}(t) y_{t},
$$

where the adjoint variable $\lambda_{r}(t)$ measures the sensitivity of the objective to changes in cumulative backstop production. Since the optimality condition for $y_{t}$ requires

$$
u^{\prime}\left(y_{t}\right)-E\left(R_{t}\right)=-\lambda_{t}(t)
$$


we have

$$
H(t)=u\left(y_{t}\right)-u^{\prime}\left(y_{t}\right) y_{t}
$$

Since $u^{\prime}\left(y_{t}\right)$ approaches $E_{\infty}, y_{t}$ and $H(t)$ approach constants. Thus, from Corollary 2 of the Appendix

$$
\Phi(T)=(1 / r) e^{-r T} H(T)=(1 / r) e^{-r T}\left[u\left(y_{T}\right)-u^{\prime}\left(y_{T}\right) y_{T}\right]
$$

Comparing (15) with the corresponding equation (7), which gives $\Phi(T)$ in the standard case, we see that $u^{\prime}\left(y_{T}\right)$ and $y_{T}$ define an equivalent constant backstop cost and production level for problem (14). That is, the solution to the first phase would not change if the backstop were assumed to have the constant cost $u^{\prime}\left(y_{T}\right)$. But this result reduces (14) to

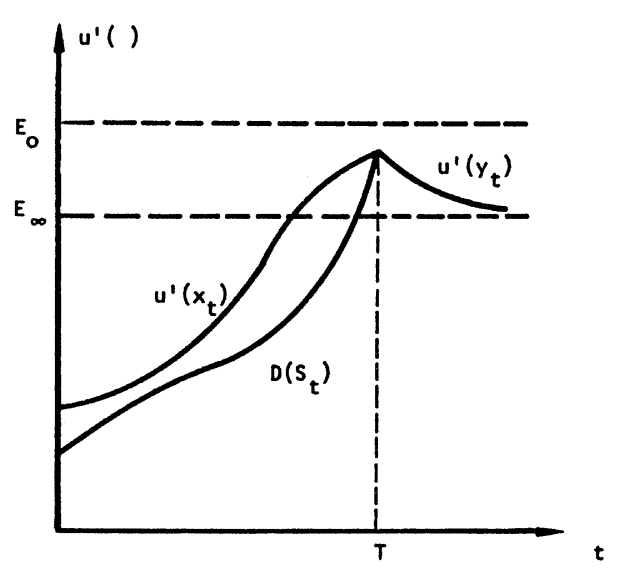

Figure 3. Learning-by-doing in the backstop.

(6): depletable energy will be extracted until $D\left(S_{T}\right)=u^{\prime}\left(y_{T}\right)$, at which point backstop production will take over. In Figure 3, we illustrate the solution to (13). Marginal utility rises on [0,T], reaching $u^{\prime}\left(y_{T}\right)=D\left(S_{T}\right)$ at $T$. Thereafter, $u^{\prime}(\cdot)$ declines toward $E_{\infty}$ as $y_{t}$ increases.

Learning-by-doing in the backstop, then, causes no change in the qualitative nature of the optimal extraction path for the depletable resource. The optimal path declines over time and marginal utility rises to the transition at $T$. Backstop production after $T$ increases over time, and marginal utility falls. But, the initial rate of production at $T$ defines an equivalent constant backstop cost and production rate; the optimal rate of production of the depletable resource at the transition date equals the initial rate of production of the backstop, and while depletable energy 
is being extracted, its marginal utility is bounded above by the equivalent backstop cost $u^{\prime}\left(y_{T}\right)$.

\section{UNCERTAIN BACKSTOP COSTS}

Backstop uncertainty has received a great deal of attention in the literature. To cite just two examples, Hoel (1978) examines the effect of uncertainty in backstop costs on optimal depletion rates, and Heal (1979) examines the impact of uncertainty on both the supply rate and the date of availability of the backstop. Our purpose in this section is to develop a simple model containing uncertain backstop costs and to analyze it using the methods developed earlier in this paper.

The simplest way to introduce uncertainty is to assume that backstop costs are given by the probability distribution $f(c)$, and that the social planner must consume all the (finite) stock of depletable energy before he can know the true value of $c$. Both Hoel and Heal work with a more complex model, in which some portion of the stock of depletable energy can be saved for extraction after the true backstop cost is revealed. The model analyzed here, although less realistic, allows for a much simpler and more suggestive analysis.

With our assumptions, we can pose the problem in a form almost identical to (6), except that the terminal reward is the expected net present value of utility from optimal backstop production, taken over the random variable $c$. Thus, we have

$$
\operatorname{Max}_{\left\{x_{t}\right\}, T} \int_{0}^{T} e^{-r t}\left[u\left(x_{t}\right)-D\left(S_{t}\right) x_{t}\right] d t+\mathscr{E}\{\Phi(T, c)\}
$$

subject to

$$
\begin{aligned}
& \dot{S}(t)=x(t) ; \quad S(0)=0, \quad S(T)=\bar{S} ; \\
& x(t) \geq 0,
\end{aligned}
$$

where

$$
\begin{aligned}
\Phi(T, c)= & \operatorname{Max}_{\left\{y_{t}\right\}} \int_{T}^{\infty} e^{-r t}\left[u\left(y_{t}\right)-c y_{t}\right] d t \\
& \text { subject to } y(t) \geq 0 .
\end{aligned}
$$

Note that the definition of $\Phi(T, c)$ is identical to the definition of $\Phi(T)$ in problem (6). Hence, by (7)

$$
\Phi(T, c)=(1 / r) e^{-r T}\left[u\left[y^{*}(c)\right]-c \cdot y^{*}(c)\right]
$$

where $y^{*}(c)$ is the solution to the equation $u^{\prime}\left(y^{*}\right)=c$.

Clearly, the conditions that determine the optimal rate of extraction of the depletable resource are identical in (17) and (6), except for the 
transversality condition, which is now

$$
H(T)=-e^{r T}(\partial \mathscr{E}\{\Phi(T, c)\} / \partial T)=\mathscr{E}\left\{u\left[y^{*}(c)\right]-c \cdot y^{*}(c)\right\} .
$$

In order to examine the effect of uncertainty in the backstop cost on the optimal depletion policy, we will compare the solution to (17) with that of (6), where the deterministic backstop cost is the mean $\bar{c}$ of $f(c)$. First, we need to establish the following result.

Proposition 5. For any probability distribution on $c$,

$$
\mathscr{E}\left\{u\left[y^{*}(c)\right]-c y^{*}(c)\right\} \geq u\left[y^{*}(\bar{c})\right]-\bar{c} y^{*}(\bar{c}) ;
$$

i.e., the expected net benefit rate of backstop production is bounded below by the net benefit rate corresponding to the expected backstop cost.

Proof. Using the chain rule, the condition $u^{\prime}\left(y^{*}\right)=c$ and the concavity of $u(\cdot)$, we obtain

$$
\frac{d^{2}}{d c^{2}}\left[u\left(y^{*}(c)\right)-c y^{*}(c)\right]=-\frac{d y^{*}(c)}{d c}=-\frac{1}{u^{\prime \prime}\left(y^{*}\right)}>0 .
$$

i.e., the function $\left[u\left(y^{*}(c)\right)-c y^{*}(c)\right]$ is strictly convex. The result then follows by Jensen's inequality (see Ferguson [1967, p. 76]).

The inequality (2) enables us to obtain the following result concerning the effect of uncertainty on the optimal depletion policy.

PROPOSITION 6. When backstop cost is uncertain with some arbitrary probability distribution, then the optimal terminal consumption rate of the depletable energy is higher than when this cost is certain at the mean value of that distribution, i.e., $x_{T}>y^{*}(\bar{c})$.

Proof. The Hamiltonian and optimality condition for (17) allows us to write

$$
H(T)=u\left(x_{T}\right)-u^{\prime}\left(x_{T}\right) x_{T} .
$$

But (19) and Proposition 5 imply that

$$
u\left(x_{T}\right)-u^{\prime}\left(x_{T}\right) x_{T} \geq u\left[y^{*}(\bar{c})\right]-\bar{c} y^{*}(\bar{c}) .
$$

Since

$$
(d / d x)\left[u(x)-u^{\prime}(x) \cdot x\right]=-x<0 \text { for } x>0,
$$

Equation 1 implies $x_{T} \geq y^{*}(\bar{c})$.

The results stated in Proposition 6 allow us to compare the production paths under conditions of certainty and uncertainty. As we noted before, 
the two problems share the same necessary conditions up to the exhaustion date. Under certainty, $x_{T}=y^{*}(\bar{c})$, and under uncertainty, $x_{T} \geq y^{*}(\bar{c})$. Thus, the entire path of production under uncertainty is higher than the path under certainty. Also, the exhaustion date must arrive sooner, since the initial resource stocks are the same.

To make this result more intuitive, we note that for any probability distribution on $c$, Equations 20 and 22 imply the existence of some "certain equivalent" backstop cost $c^{*}$ satisfying

$$
0<c^{*} \leq \bar{c} \text { and } \mathscr{E}\left\{u\left[y^{*}(c)\right]-c y^{*}(c)\right\}=u\left[y^{*}\left(c^{*}\right)\right]-c^{*} y^{*}\left(c^{*}\right) .
$$

Substituting (23) into (19) makes the necessary conditions for problem (17) identical to the ones for problem (6) with backstop cost $c^{*}$.

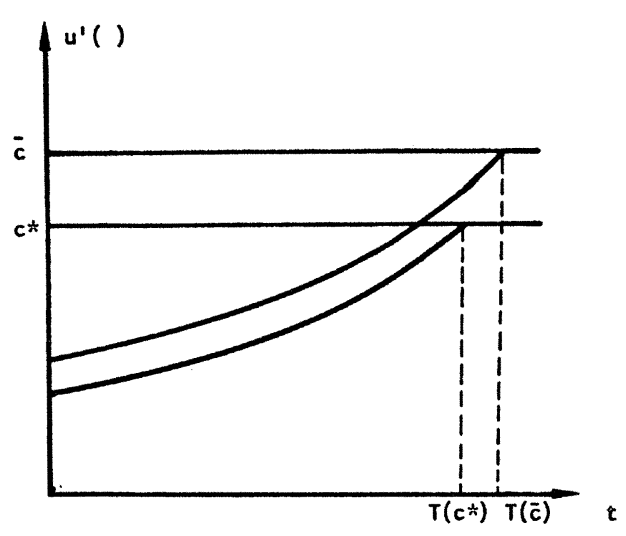

Figure 4. Uncertain backstop cost.

It is possible, therefore, to replace the uncertain problem (17) with a certain problem in which the backstop cost is $c^{*}$. Consequently, in the presence of uncertainty about the backstop cost, it is optimal for the social planner to act as if that cost is lower than its expected value. This situation will have the effect of raising the depletion rate $x_{t}$, which in turn lowers $u^{\prime}\left(x_{t}\right)$ and moves the exhaustion date up from $T(\bar{c})$ to $T\left(c^{*}\right)$. Figure 4 shows the path of $u^{\prime}(\cdot)$ to $T(\bar{c})$ under certainty, and the path of $u^{\prime}(\cdot)$ to $T\left(c^{*}\right)$ under uncertainty. At $T\left(c^{*}\right)$ the true value of $c$ is revealed. Up to this point, the planner has been acting as if $c$ would turn out to be $c^{*}$, which is less than the mean of $f(c)$. But if, for the sake of argument, $f(c)$ was symmetric about $\bar{c}$, the actual value of $c$ revealed at $T\left(c^{*}\right)$ would exceed $c^{*}$ with a probability greater than 0.5 . Thus, in a sense the planner must expect the backstop cost to exceed $c^{*}$, and consequently the optimal 
level of marginal utility $u^{\prime}[y(c)]$ to exceed $c^{*}$. This situation implies that an upward jump at $T$ in marginal utility, or a downward jump in the production rate, is inherent in the solution to this problem.

\section{SUMMARY}

In this paper we have developed a simple method for analyzing a class of problems in the theory of resource economics. The method rests on the fact that, in certain problems with two resources, it is optimal to produce from one resource first, and then from the other. This two-phase property allows us to replace an infinite-horizon optimal control problem that has two control variables with a free-end time finite-horizon problem that has one control variable. This procedure is simpler and more general than the one now in use. It allows us to derive Heal's theorem in a straightforward way. It also allows us to fully solve several complex resource problems: one in which the backstop technology is characterized by declining costs, and another where the cost of the backstop is uncertain.

\section{APPENDIX}

In this appendix we prove a theorem and two corollaries concerning the optimal value of a general control problem with discounted objective.

Theorem A.1. Let $J^{*}$ be the optimal value of the objective function for the control problem

$$
\begin{aligned}
& \operatorname{Max}_{\{u(t)\}} \int_{t_{1}}^{t_{2}} e^{-r t} L(x(t), u(t), t) d t \\
& \text { subject to } \dot{x}(t)=f(x(t), u(t), t) .
\end{aligned}
$$

Then

$$
J^{*}=\frac{1}{r}\left[e^{-r t_{1}} H\left(t_{1}\right)-e^{-r t_{2}} H\left(t_{2}\right)\right]+\frac{1}{r} \int_{t_{1}}^{t_{2}} e^{-r t} \frac{\partial H}{\partial t} d t .
$$

Proof. First-order necessary conditions for this problem can be derived from the current-value Hamiltonian:

$$
H=L(x(t), u(t), t)+\lambda(t) \cdot f(x(t), u(t), t) .
$$

The state-control pair $x(t), u(t)$ must satisfy

$$
\partial H / \partial u=0
$$

and

$$
\dot{x}(t)=f(x(t), u(t), t)
$$


while the adjoint variable $\lambda(t)$ obeys

$$
\dot{\lambda}(t)=r \lambda(t)-\partial H / \partial x .
$$

Along the optimal trajectory, we have

$$
\begin{aligned}
\int_{t_{1}}^{t_{2}} e^{-r t} L(x(t), u(t), t) d t \\
=\int_{t_{1}}^{t_{2}} e^{-r t}[L(x(t), u(t), t)+\lambda(t)[f-\dot{x}]] d t \\
=\int_{t_{1}}^{t_{2}} e^{-r t}[H-\lambda(t) \dot{x}] d t .
\end{aligned}
$$

Integrating by parts, we obtain

$$
\int_{t_{1}}^{t_{2}} e^{-r t} H d t=-\left.\frac{1}{r} e^{-r t} H\right|_{t_{1}} ^{t_{2}}+\frac{1}{r} \int_{t_{1}}^{t_{2}} e^{-r t} \frac{d H}{d t} d t .
$$

Substituting (A.6) into (A.5) then gives

$$
\begin{aligned}
\int_{t_{1}}^{t_{2}} e^{-r t}[L+\lambda(t) & (f-\dot{x})] d t \\
& =-\left.\frac{1}{r} e^{-r t} H\right|_{t_{1}} ^{t_{2}}+\int e^{-r t}\left[\frac{1}{r} \frac{d H}{d t}-\lambda(t) \dot{x}\right] d t .
\end{aligned}
$$

Note that $H$ is a function of $x(t), u(t), \lambda(t)$, and (in general) $t$ itself. Thus,

$$
d H / d t=(\partial H / \partial x) \dot{x}+(\partial H / \partial u) \dot{u}+(\partial H / \partial \lambda) \dot{\lambda}+\partial H / \partial t .
$$

But from (A.2) and (A.4) and $\partial H / \partial x=\dot{x}$, we have

Thus,

$$
d H / d t=[r \lambda(t)-\dot{\lambda}(t)] \dot{x}+\dot{x} \dot{\lambda}+\partial H / \partial t .
$$

$$
\begin{aligned}
& \int_{t_{1}}^{t_{1}} e^{-r t}\left[\frac{1}{r} \frac{d H}{d t}-\lambda(t) \dot{x}\right] d t \\
& =\int_{t_{1}}^{t_{2}} e^{-r t}\left[\lambda(t) \dot{x}-\frac{1}{r} \dot{x} \dot{\lambda}(t)-\frac{1}{r} \dot{\lambda}(t) \dot{x}-\lambda(t) \dot{x}+\frac{1}{r} \frac{\partial H}{\partial t}\right] d t \\
& =\frac{1}{r} \int_{t_{1}}^{t_{2}} e^{-r t} \frac{\partial H}{\partial t} d t .
\end{aligned}
$$


Substituting (A.10) in (A.7) yields the theorem:

$$
\begin{aligned}
& \int_{t_{1}}^{t_{2}} e^{-r t}[L+\lambda(t)(f-\dot{x})] d t \\
&=-\left.\frac{1}{r} e^{-r t} H\right|_{t_{1}} ^{t_{1}}+\frac{1}{r} \int_{t_{1}}^{t_{2}} e^{-r t} \frac{\partial H}{\partial t} d t .
\end{aligned}
$$

The following corollaries are direct consequences of the theorem.

CoRollaRY 1. If the Hamiltonian for $\mathrm{P} 1$ is not a direct function of time (i.e., $\partial H / \partial t=0$ ), then

$$
J^{*}=(1 / r)\left[e^{-r t_{1}} H\left(t_{1}\right)-e^{-r t_{2}} H\left(t_{2}\right)\right] .
$$

Corollary 2. If the upper limit $t_{2}$ in $\mathrm{P} 1$ is $+\infty$, and the Hamiltonian approach a constant as $t \rightarrow \infty$, then

$$
J^{*}=(1 / r) e^{-r t_{1}} H\left(t_{1}\right) .
$$

\section{REFERENCES}

Clark, R. A. 1978. The Relationship between Price and Marginal Extraction Cost for a Resource with a Backstop Technology: Comment. Bell J. Econ. (Spring).

Ferguson, T. 1967. Mathematical Statistics. Academic Press, New York.

Hanson, D. A. 1980. Increasing Extraction Costs and Resource Prices: Some Further Results. Bell. J. Econ. (Spring).

HEaL, G. 1976. The Relationship between Price and Extraction Cost for a Resource with a Backstop Technology. Bell J. Econ. (Autumn).

HeAL, G. 1979. Uncertainty and the Optimal Supply Policy for an Exhaustible Resource. In Advances in the Economics of Energy and Resources, Vol. 2. Jai Press, Greenwich, Conn.

HoEL, M. 1978. Resource Extraction when a Future Substitute Has an Uncertain Cost. Rev. Econ. Studies (October).

Kemp, M. C., AND N. V. LoNG. 1980. On Two Folk Theorems Concerning the Extraction of Exhaustible Resources. Econometrica 48, No. 3 (April).

Powell, S. 1983. The Transition to Nondepletable Energy. Ph.D. dissertation, Engineering-Economic Systems, Stanford University. 